\section{Review: vaccines against human papillomavirus prevent cervical cancer precursors in young women}

\section{QUESTION}

Do vaccines against human papillomavirus (HPV) prevent cervical cancer precursors in young women?

\section{METHODS}

Data sources: Medline and EMBASE/Excerpta Medica (to May 2007); Medline in process (to June 2007); Cochrane Library and Cochrane Central Register of Controlled Trials (Issue 1, 2007); conference proceedings (2004-7); clinical trial registers; Google Scholar; public health announcements; reference lists; and vaccine manufacturers.

Study selection and assessment: randomised controlled trials (RCTs) that compared a vaccine against $\geqslant 1$ oncogenic type of HPV with placebo or no HPV vaccine for prophylaxis of oncogenic HPV-related infection and disease in women. 9 reports of 6 RCTs ( $n=40323$; mean age $20 \mathrm{y}$, range 15-26 y) met the selection criteria. 1 RCT $(n=2392)$ evaluated a monovalent HPV 16 vaccine, 2 RCTs ( $\mathrm{n}=19$ 757) evaluated a bivalent HPV 16/18 vaccine, and 3 RCTs ( $n=18$ 174) evaluated a quadrivalent HPV 6/11/16/18 vaccine. Quality assessment of individual trials was based on the Jadad scale and allocation concealment; all 6 RCTs were of high methodological quality.
Outcomes: high-grade cervical lesions (high-grade squamous intraepithelial lesion or grade 2 or 3 cervical intraepithelial neoplasia [CIN]), any grade cervical lesions, persistent HPV infection, external genital lesions, and adverse events.

\section{MAIN RESULTS}

HPV vaccines reduced the risks of all outcomes in the modified intention-to-treat population that included 84$100 \%$ of randomised women (table). Groups did not differ for serious adverse events (table).

\section{CONCLUSION}

Vaccines against human papillomavirus (HPV) in young women prevent persistent infection and precancerous cervical disease associated with vaccine-specific HPV types.

\section{ABSTRACTED FROM}

Rambout L, Hopkins L, Hutton B, et al. Prophylactic vaccination against human papillomavirus infection and disease in women: a systematic review of randomized controlled trials. CMAJ 2007;177:469-79.

Correspondence to: Ms L Rambout, Ottawa Hospital, Ottawa, Ontario, Canada; Irambout@ottawahospital.on.ca

Source of funding: not stated.

- Clinical impact ratings: Family/General practice 5/7; Infectious disease 5/7; Oncology 5/7; Women's health 6/7

Vaccines against oncogenic types of human papillomavirus (HPV) $v$ placebo or no HPV vaccine to prevent vaccine-type HPV-associated cervical cancer precursors in young women (modified intention-to-treat population)*

\begin{tabular}{|c|c|c|c|c|c|}
\hline \multirow[b]{2}{*}{ Outcomes at $15-60$ months } & \multirow[b]{2}{*}{ Number of trials (n) } & \multicolumn{2}{|c|}{ Weighted event rates } & \multirow[b]{2}{*}{$\operatorname{RRR}(95 \% \mathrm{CI})$} & \multirow[b]{2}{*}{ NNT (Cl) } \\
\hline & & Vaccine & Control & & \\
\hline Grade 2 CIN or worse & $5(36266)$ & $0.8 \%$ & $1.5 \%$ & $48 \%$ (37 to 57$)$ & 138 (116 to 179$)$ \\
\hline Any grade $\mathrm{CIN}$ & $5(24613)$ & $0.7 \%$ & $1.9 \%$ & $64 \%(55$ to 71$)$ & 81 (73 to 95$)$ \\
\hline Persistent HPV infection at 6 months & $3(14207)$ & $0.9 \%$ & $4.0 \%$ & $77 \%(72$ to 81$)$ & 33 (31 to 35$)$ \\
\hline Persistent HPV infection at 12 months & $2(7774)$ & $0.4 \%$ & $1.6 \%$ & $74 \%$ (59 to 84$)$ & 86 (76 to 108$)$ \\
\hline External genital lesions & $2(5981)$ & $1.1 \%$ & $3.5 \%$ & $69 \%$ (56 to 77$)$ & 41 (37 to 51$)$ \\
\hline Serious adverse events & $6(39609)$ & $2.2 \%$ & $2.2 \%$ & $0 \%(-14$ to 13$)$ & Not significant \\
\hline
\end{tabular}

${ }^{*} \mathrm{CIN}=$ cervical intraepithelial neoplasia; other abbreviations defined in glossary. Weighted event rates, RRR, NNT, and Cl calculated from data in article.

T he systematic review by Rambout et al is a high-quality synthesis of the evidence to date on the effectiveness of prophylactic HPV vaccination in preventing persistent HPV infection and precancerous cervical changes in young women. Infection with HPV is common; ${ }^{1}$ however, current primary prevention strategies, such as condom use, can only reduce the risk of transmission. ${ }^{2}$ The vaccines included in this review provide highly effective protection against 2 high-risk HPV types (16 and 18), which account for $70 \%$ of all cervical cancers. 1 of the vaccines tested provides additional coverage against non-oncogenic HPV types 6 and 11, which have been associated with the development of external genital disease.

Questions remain about the long-term effectiveness of the vaccine in reducing cervical cancer incidence and mortality, effectiveness of the vaccine in real world populations, duration of effect, and the optimal age to immunise young women if the vaccine is publicly funded. Despite these gaps in knowledge, it is evident from the results of this review that prophylactic HPV vaccination can prevent typespecific HPV infection and precancerous cervical lesions in healthy young women (15-26 y of age) who receive all 3 doses of the vaccine and have not been previously infected with these strains.

The conclusions of the review by Rambout et al are relevant to nurses working in public health and primary health care. Education should be provided to the public about HPV. Nurses can use the results of this review to help parents and young women understand the risks and benefits of immunisation and make informed decisions. It is essential that clients understand that the vaccines do not protect against all strains of HPV and that long-term vaccine efficacy has not been determined. Vaccination must be accompanied by education on the importance of continued routine cervical screening and safer sex practices, regardless of immunisation status. HPV vaccines provide a new and promising strategy for primary prevention of cervical cancer that should be accessible to all women who can benefit from them.

\section{Jacqueline Muresan, RN, BScN}

MSc student

McMaster University

Hamilton, Ontario, Canada

1. National Advisory Committee on Immunization Statement on human papillomavirus vaccine. Can Commun Dis Rep 2007;33(ACS-2):1-31.

2. Manhart LE, Koutsky LA. Do condoms prevent genital HPV infection, external genital warts, or cervical neoplasia? A meta-analysis. Sex Transm Dis 2002;29 725-35 